\title{
In Situ
}

Revue des patrimoines

$32 \mid 2017$

Le collectif à l'œuvre. Collaborations entre architectes et plasticiens (XXe-XXle siècles)

\section{« Expérience Nîmes ». Un projet de gestion urbaine croisant architecture, art et design (1983-1995)}

The Nîmes experiment, a project for urban development bringing together architecture, art and design (1983-1995)

\section{Fanny Bocklandt}

\section{OpenEdition}

\section{Journals}

Édition électronique

URL : http://journals.openedition.org/insitu/15074

DOI : $10.4000 /$ insitu. 15074

ISSN : 1630-7305

\section{Éditeur}

Ministère de la Culture

\section{Référence électronique}

Fanny Bocklandt, « «Expérience Nîmes ». Un projet de gestion urbaine croisant architecture, art et design (1983-1995) », In Situ [En ligne], 32 | 2017, mis en ligne le 03 octobre 2019, consulté le 03 octobre 2019. URL : http://journals.openedition.org/insitu/15074 ; DOI : 10.4000/insitu.15074

Ce document a été généré automatiquement le 3 octobre 2019.

\section{(i) $\odot$

In Situ Revues des patrimoines est mis à disposition selon les termes de la licence Creative Commons Attribution - Pas d'Utilisation Commerciale - Pas de Modification 4.0 International. 


\section{« Expérience Nîmes ». Un projet de gestion urbaine croisant architecture, art et design (1983-1995)}

The Nîmes experiment, a project for urban development bringing together architecture, art and design (1983-1995)

\section{Fanny Bocklandt}

J'adresse des remerciements particuliers à Madame Éléonore Marantz-Jaen ainsi qu'à Madame Delphine Bière, pour la confiance et les relectures attentives qu'elles m'ont apporté tout au long de la rédaction de cet article.

Le troisième millénaire est là, ou presque. J'imagine, si proche, l'an 2000, et Nîmes, nouvelle plate-forme internationale. Nîmes a gardé ses vestiges, mais s'est réinventée une histoire, qui mêle harmonieusement, sous le soleil, les industriels et les créatifs, les chercheurs et les

sportifs, les commerçants, les touristes, les boulistes, les familles, les enfants ... C'est simple,

Nîmes revit. Elle a reconquis sa prospérité, retrouvé une identité, développé sa spécificité, recouvré un mieux-vivre. On visite toujours les arènes, mais le nouveau Nîmes intrigue, suscite des passions. Prestigieuse, spectaculaire, unique, réalisée avec les créateurs nationaux et internationaux qui se sont ralliés à mon projet, Nîmes est devenue la capitale du Design et de

l'Architecture'. Jean Bousquet, 1986 


\section{Nîmes (1983-1995), une ville en mouvement}

Dès le milieu des années 1970, les bouleversements consécutifs à la transition postindustrielle conduisent les collectivités locales à redéfinir leurs politiques urbaines. En la matière, les plus précoces sont souvent celles qui subissent le plus durement la crise industrielle ${ }^{2}$. Face à la montée du chômage, à la fuite des capitaux ou à la constitution de vastes friches sur les anciennes emprises industrielles, ces municipalités mettent en œuvre des actions destinées à renouveler le tissu économique et urbain. Pour rendre les territoires attractifs, la capacité à attirer (et à maintenir) les entreprises constitue un enjeu majeur des politiques locales. Les municipalités, soutenues par les récentes lois de décentralisation, exercent dorénavant des compétences détenues jusqu'alors par le pouvoir central et ses administrations ${ }^{3}$. Dans un contexte de compétition territoriale, les modes de gestion urbaine se modifient sous l'influence de cette quête d'attractivitét. L'allègement de la fiscalité, l'amélioration de l'accessibilité à travers le développement des réseaux de transports et la constitution d'un parc immobilier adapté au secteur tertiaire sont souvent les premières initiatives engagées en faveur des entreprises. Pour convaincre les sociétés à haute valeur ajoutée, notamment celles qui emploient des intellectuels ou des créatifs, de venir s'installer dans des villes dont l'économie est en déclin, la qualité du cadre de vie et la vie culturelle sont investies et valorisées. Elles seront bientôt mises en scène, généralement dans le cadre de grands projets urbains.

[En 1981,] François Mitterrand, choisissant Jack Lang, faisait le pari de la culture. C'était un domaine, plus encore probablement que d'autres, réduit à sa plus simple expression, sclérosé, décadent. Il est vrai que l'héritage des décennies précédentes était plutôt maigre. Mais les objectifs du nouveau gouvernement étaient clairs : redonner à la France dans le domaine des arts, dans celui de l'économie comme dans celui de la recherche le goût de l'initiative, le goût de la création, le goût de la compétitivité

2 rappelle très justement Yves Hayat ${ }^{5}$. Sans tarder, les maires et présidents de conseils généraux ou régionaux, de Montpellier à Lille, de Lyon à Nantes en passant par Tours, tous ont leurs grands projets ${ }^{6}$, à l'instar de Jean Bousquet à Nîmes.

3 Au cours de l'année 1983, année de l'élection de Jean Bousquet au fauteuil de maire, dans ce contexte particulier, la Ville de Nîmes, préfecture du Gard, fait ainsi le choix d'enclencher un processus de transformation urbaine qui se prolonge jusqu'en 1995 (fig. 1). Cette expérience fait l'objet d'une vive attention et, si elle intéresse aussi bien les architectes que les urbanistes, les personnalités politiques, les sociologues, les designers ou les artistes au plan national, c'est parce que l'équipe municipale en place se confronte à des problématiques importantes, partagées par beaucoup d'acteurs de la ville au cours du dernier quart du $\mathrm{xx}^{\mathrm{e}}$ siècle : comment construire dans un tissu urbain historique ? Comment protéger le patrimoine naturel et bâti et créer des conditions de vie agréables? Comment trouver des solutions aux problèmes des banlieues et des périphéries de façon générale ? Comment anticiper l'évolution de la ville? 
Figure 1

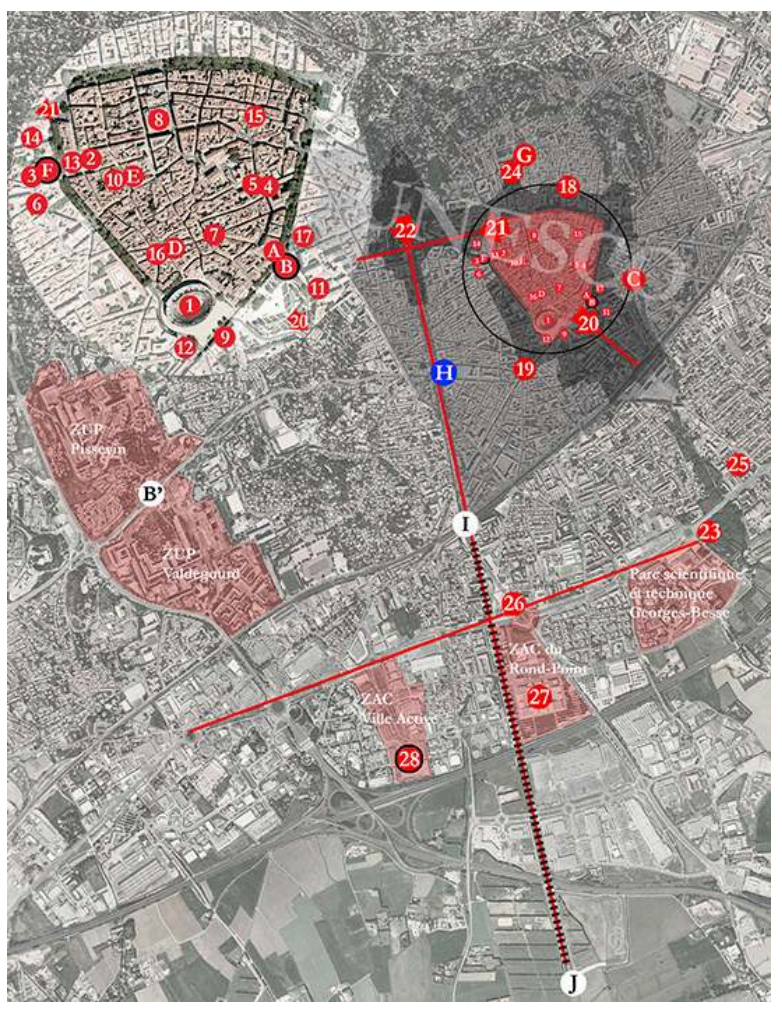

Plan de repérage.

Phot. Fanny Bocklandt, 2017. (c) Fanny Bocklandt.

\section{Un projet urbain placé sous le signe de la création}

Ces questions sous-tendent un projet urbain enclenché en 1983 et formalisé en 1991 sous le nom d'«Expérience Nîmes: vers une ville sans banlieue ${ }^{7}$ " (fig. 2). De cette démarche originale qui ambitionnait de créer «les conditions d'un développement urbain global de qualité ${ }^{8} "$, la plupart des observateurs retiennent une initiative individuelle, voire individualiste, impulsée par le maire de l'époque Jean Bousquet (né en 1932). En effet, élu en mars 1983 pour le parti de l'Union démocrate française (UDF) - plus tard candidat aux élections législatives de mars 1986 - Jean Bousquet apparaît comme un homme politique d'un nouveau genre. Originaire de Nîmes, fondateur de la maison de prêt-à-porter $\mathrm{Cacharel}^{9}$, il a toujours été passionné par l'art. Il est à l'époque un chef d'entreprise en pleine réussite qui, en quelques années, a hissé sa société aux premiers rangs des industries textiles en France avec un chiffre d'affaires avoisinant 400 millions de francs (valeur 1986) ${ }^{10}$. 


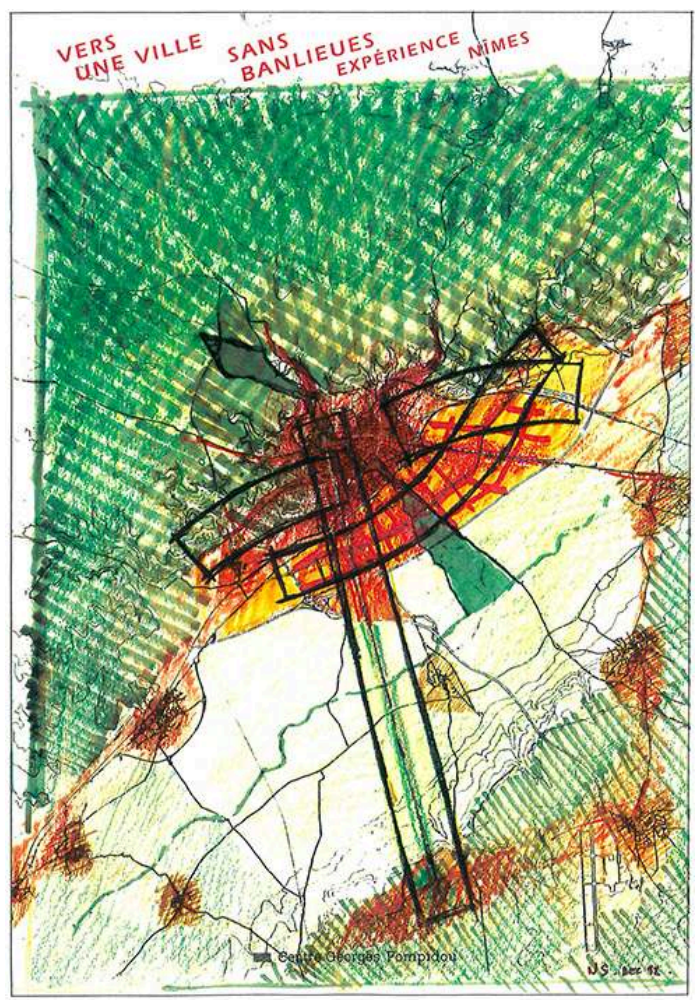

Plan d'ordonnancement (décembre 1992) ayant servi d'affiche à l'exposition « Vers une ville sans banlieues. Expérience Nîmes » (Paris, Centre Pompidou, 10 février-10 juillet 1993), coll. Agence d'urbanisme de Nîmes.

(C) Agence d'urbanisme de Nîmes.

5 Il entend gérer sa ville natale avec la rigueur et le dynamisme créateur d'un chef d'entreprise. Partisan d'une politique touristico-culturelle de « qualité », Jean Bousquet a, entre autres, impulsé l'ouverture d'une galerie municipale d'art contemporain (1984), accueilli la donation Lintas dans la chapelle des Jésuites (1986) (voir fig. 1 réf. 4), créé une biennale internationale de l'affiche (1986), invité l'Opéra de Paris dans les arènes de Nîmes (1989) ou coproduit de grands spectacles avec Bercy. Parallèlement à ces initiatives en faveur du développement culturel, il souhaite également faire rayonner la ville hors des frontières de l'Hexagone au travers de l'architecture et de l'urbanisme: «L'architecture est une préoccupation de l'époque [...]. Toutes les villes s'aperçoivent qu'elles ont eu un trou pendant 20 ou 30 ans, alors que l'urbanisme a toujours été une force dans notre pays. Nous devons continuer à investir dans ce domaine $»^{11}$, affirme-t-il en 1989 lors d'un entretien pour le magazine régional Architecture Méditerranéenne ${ }^{12}$. En effet, avec ses arènes et sa Maison Carrée (voir fig. 1 réf. 1 et 2), Nîmes possède un patrimoine monumental renvoyant à une histoire bimillénaire et, fort de cet héritage, Jean Bousquet entend désormais promouvoir l'art contemporain :

Ce qui m'intéresse, déclare-t-il, c'est d'affronter les différentes époques. [...] Il est passionnant de confronter les héritages culturels et les exigences de la vie moderne. Mener une action culturelle cohérente, ce n'est pas uniquement conserver le patrimoine. C'est, au contraire, associer ce patrimoine aux créations les plus novatrices. 
Il considère que l'artiste qui investit un contexte historique ne doit pas avoir de projet prédéterminé mais « établir un dialogue spirituel avec l'âme du site ${ }^{13}$ ». Dans son esprit, les interventions artistiques qu'il souhaite initier permettront de promouvoir l'idée d'une interaction possible entre les monuments historiques - soit un héritage prestigieux - et des aménagements contemporains qu'il souhaite inscrire dans cette histoire ${ }^{14}$. Une cinquantaine de projets à vocation artistique ou de commandes et interventions artistiques (au sens large du terme) sont engagés au cours des deux mandatures de Jean Bousquet, dans les seules limites administratives de la cité: restauration d'édifices patrimoniaux tels que l'hôtel Rivet (1987, arch.: Ariel Balmassiere, Jean-Michel Wilmotte, artistes : Alain Jeanmet; Léopold Gest ; Bernard Pagès) (voir fig. 1 - réf. 5) ou l'hôtel de ville (1990, arch.: Ariel Balmassiere, JeanMichel Wilmotte, Norman Foster, designer : Philippe Starck) (voir fig. 1 - réf. 7) par des équipes pluridisciplinaires composées de décorateur, styliste d'intérieur, sculpteur, architecte et designer; reconversion du fort Vauban (1995, arch.: Andréa Bruno, artistes: Erik Dietman) (voir fig. 1 - réf. 24) en université15; construction d'une nouvelle médiathèque et centre d'art contemporain ${ }^{16}$ - le Carré d'art (1993, arch.: Norman Foster) (voir fig. 1 - réf. 3) - par l'un des grands noms de l'architecture mondiale de l'époque, Norman Foster (né en 1935), ou encore de l'ensemble d'habitation HLM Nemausus ${ }^{17}$ (1986, arch. : Jean Nouvel) (voir fig. 1 - réf. 25) par un jeune loup de la scène française, Jean Nouvel (né en 1945), qui y impose « $25 \mathrm{~m}^{2} \mathrm{~d}$ 'art par cellule $\aleph^{18}$. Les collaborations entre les différents acteurs intervenant dans la fabrique de la ville de Nîmes font l'objet d'une vive attention de la part de la profession, ce qui conduit le critique d'architecture Patrice Goulet à déclarer que «depuis 1983, le monde des architectes, designers et artistes de renom, semble participer d'une véritable "ruée vers Nîmes" "19. Cette effervescence vaut bientôt à la ville d'être qualifiée de «nouvelle capitale du design et de l'architecture ${ }^{20}$ ». Si les projets initiés par Jean Bousquet ont pour objectif principal de relancer l'économie de la ville, ils concourent à faire émerger l'image plus générique d'une ville dynamique et créative. De cette époque, l'histoire retient surtout le «Carré d'art " (fig. 3), souvent présenté comme la "réplique nîmoise» du Centre Beaubourg ou encore l'atelier de design industriel labellisé "De Nîmes" (voir fig. 1 - réf. 28) dont les chefs de file sont le designer Philippe Starck (né en 1949) et l'architecte Jean Nouvel ${ }^{21}$. Cependant, un autre projet, une entreprise de collaboration artistique méconnue du grand public, polarise les enjeux de la politique de gestion urbaine de la Ville, le "Centre d'expérimentation de Nîmes ", qui apparaît comme un laboratoire expérimental de l'urbain. 


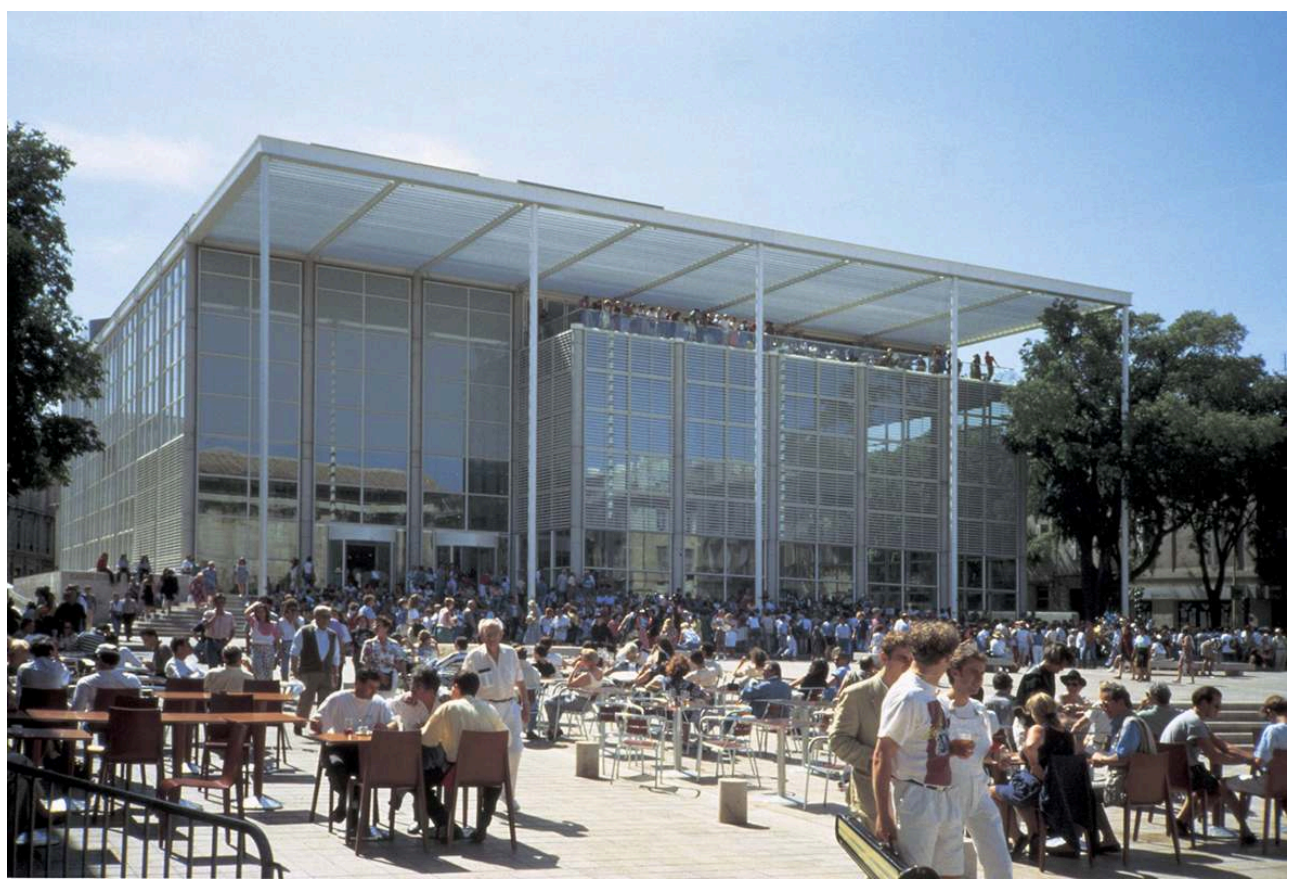

Carré d'art - Médiathèque de Nîmes (arch. : Foster and Partners, 1993).

PHOT. FOSTER AND PARTNERS, 1993. ( ) FOSTER AND PARTNERS.

\section{Le «Centre d'expérimentation de Nîmes ", laboratoire conceptuel?}

7 Dès le début des années 1990, forts de leurs expériences personnelles et de leurs convictions, les acteurs du projet "Expérience Nîmes », hommes politiques, urbanistes, architectes, designers ou artistes, établissent plusieurs constats. À l'aune des réflexions induites par le projet urbain mis en œuvre depuis l'élection de Jean Bousquet en 1983, ils souhaitent que soit créé un « laboratoire expérimental $»^{22}$ qui, à la fois, favoriserait une réflexion théorique sur la ville mais aussi, tendrait, sur le plan opérationnel, vers une "qualité totale» en matière d'urbanisme. Ce laboratoire expérimental doit prendre appui sur des références culturelles et formelles qui « remettent à l'honneur des notions empruntées à la ville traditionnelle, ignorées par l'urbanisme fonctionnaliste qui a fait table rase de l'épaisseur historique des villes $»^{23}$. Il doit permettre de retrouver une « qualité des espaces urbains et des pratiques de la ville [...] un art urbain $»^{24}$. La démarche des décideurs et des aménageurs nîmois ${ }^{25}$ s'appuie donc sur la volonté de faire revivre un idéal social dont les principes fondateurs renvoient au Bauhaus ou au mouvement De Stijl. En effet, dans les années 1930, alors que le Bauhaus tentait de réconcilier l'art et la technique dans le but de créer un environnement urbain de qualité, Piet Mondrian, animateur du mouvement hollandais De Stijl, déclarait que

beauté et harmonie [étaient] devenues un idéal irréalisable : réfugiées dans l'art, elles ont été expulsées de la vie et de l'environnement. Demain, au contraire, la réalisation de l'équilibre plastique dans la réalité sensible de notre environnement remplacera l'œuvre d'art. Alors, nous n'aurons plus besoin de peintures, ni de sculptures, puisque nous vivrons au milieu d'un art réaliséé 


\section{d'environnement redéfinit leur discipline, le terme d'« école ", souvent employé pour} désigner ces mouvements d'avant-garde, est en substance très important parce qu'il «traduit un courant de pensée sur la ville devenant dès lors l'expression de l'existence d'une culture urbaine marquante dans la société $»^{27}$. À l'aube du XxI siècle, au vu du rôle considérable que jouent les structures et les hommes à l'intérieur des phénomènes de croissance urbaine, il semble désormais plus que nécessaire aux aménageurs nîmois de mettre au point des stratégies capables d'en permettre la maîtrise. La nouvelle «école » de pensée nîmoise se développe sur la base de trois principes. Le premier d'entre eux est de permettre une plus grande liberté de création. En effet, si pour les responsables urbains nîmois, la profession d'architecte est alors globalement sinistrée, ils considèrent aussi que les autres spécialistes de la ville s'enferment dans une technicité qui, pour utile et respectable qu'elle soit, ne permet pas d'assumer la véritable portée des décisions auxquelles ils participent (ou tout au moins qu'ils suggèrent, étudient et défendent). Cette opinion est d'ailleurs partagée par une grande partie de la profession et de la critique ${ }^{28}$. Il en résulte que, sur le terrain professionnel, la programmation technique tend désormais à se substituer à la responsabilité éthique du projet urbain. Jean Bousquet met en avant que la liberté de création est ce qui importe le plus, qu'il «faut laisser prendre des risques aux créateurs, beaucoup de risques, et leur donner pleinement le droit à l'erreur $»^{29}$. Ainsi, dans un premier temps, il souhaite que les concours et consultations visant à sélectionner les intervenants, qu'ils soient architectes, artistes ou designers, soient systématiques et obligatoires, gages selon lui d'une libre expression de la création «trop souvent enfermée dans un corset de règles paralysantes ${ }^{30}$ \%. De même, Jean Bousquet estime nécessaire de favoriser et d'organiser la rencontre - voire la collaboration - entre les différents professionnels intervenant sur la ville, notamment entre les architectes et les artistes. Il est convaincu que ces interactions sont potentiellement créatrices et peuvent faire émerger une vision renouvelée de la ville du présent et du futur ${ }^{31}$. Pour les responsables nîmois, la créativité doit désormais dépasser le seul édifice à construire pour concerner l'environnement, la ville dans son ensemble ${ }^{32}$. Les urbanistes Joseph Juvin ${ }^{33}$ (né en 1936) et Nicolas Soulier ${ }^{34}$ (né en 1951), responsables en chef du projet Expérience Nîmes, mettent en avant que «l'espace urbain d'une ville peut assez aisément se découper en plusieurs secteurs géographiques sur lesquels des équipes différentes peuvent intervenir $»^{35}$. De cette confrontation fructueuse entre différentes équipes, ils attendent qu'émerge "un projet urbain global de qualité36", préférable selon eux à une conception unitaire.

9 La qualité qu'ambitionnent d'atteindre les responsables urbains nîmois par la collaboration implique par conséquent de nouvelles méthodes de travail qui, en englobant toutes les disciplines et en les recomposant spatialement, permettent d'échapper à la médiocrité d'une conception « réductrice, trop souvent répétitive [...] et vouée à l'échec ou à l'erreur ${ }^{37}$ ». Cependant, notons que si cette ambition initiale concerne tout autant le centre historique de Nîmes que sa périphérie, la tâche à accomplir dans ces différents secteurs diffère tant le centre est restreint et la périphérie immense ${ }^{38}$. Seul le fait de décupler le nombre d'équipes de conception, de leur donner les moyens de définir, piloter et coordonner leurs études permettrait de traiter l'ensemble de territoire. Si Jean Bousquet n'y est pas hostile sur le principe, il estime que « le nombre de professionnels qualifiés [nîmois] est nettement insuffisant à 
ce jour pour faire face à la demande, et [que] l'état de la recherche expérimentale sur cette question est à faire progresser $\aleph^{39}$. Il entend donc s'appuyer sur les forces en présence, mais aussi attirer des "équipes d'accompagnement " extérieures ${ }^{40}$. La mise en œuvre du projet consiste donc, dans un premier temps, à renforcer l'attractivité de la ville auprès des professionnels de l'urbain mais aussi des chercheurs, des étudiants et des enseignants qui pourraient, par leurs travaux, participer à la connaissance (et à la reconnaissance) du processus de transformation urbaine qu'il a impulsé, dans le cadre du « Centre d'expérimentation de Nîmes » créé en 1991. Dans cette véritable entreprise médiatique, voire de validation intellectuelle par l'analyse et la théorisation du fait urbain, il est par exemple prévu de soutenir la publication des travaux scientifiques et d'offrir aux chercheurs ayant "réalisé les travaux les plus remarquables " ${ }^{41}$, l'opportunité d'intégrer le réseau de professionnels intervenants alors à Nîmes. La capitalisation des actions engagées doit permettre, à terme, l'émergence "d'une réflexion globale sur la ville et peut-être d'un courant de pensée ce qui, en France, fait à ce jour gravement défaut pour guider les actions quotidiennes ${ }^{42}$.

\section{Comment œuvrer ensemble?}

Si les ambitions conceptuelles du "Centre d'expérimentation de Nîmes » ne seront pas totalement atteintes, une première application de son socle théorique prend corps et se vérifie au travers des actions entreprises entre 1991 et 1995 dans le cadre de la politique urbaine impulsée par Jean Bousquet et les responsables des services de l'urbanisme de la ville. Au cours des décennies précédentes, la conception et la gestion des espaces publics étaient restées cantonnées à une approche essentiellement fonctionnelle, du fait notamment du développement extrêmement rapide de la ville ${ }^{43}$. La gestion des flux automobiles, tout comme les problématiques liées au stationnement des véhicules, avaient prévalu au détriment de la qualité du «cadre de vie $»^{44}$. L'application de normes faisant peu de cas de la qualité urbaine, l'espace public était sorti « amoindri dans son rôle de lieu de mémoire et d'échanges, pensé désormais en négatif par rapport à des formes bâties répondant souvent aux intérêts individuels des promoteurs $»^{45}$. Conscients que l'espace public était pourtant la principale expression de la vie sociale ${ }^{46}$ et que "la qualité urbaine [pouvait] entraîner l'émotion ${ }^{47}$, Joseph Juvin et Nicolas Soulier placent la question de l'espace public à Nîmes au centre des politiques d'aménagement. Elle constitue même la base d'une réflexion globale qui s'engage sur la ville et son avenir à l'aube du $\mathrm{xxI}^{\mathrm{e}}$ siècle. Les urbanistes définissent en effet une échelle d'intervention dans laquelle l'espace public devient un "générateur " de formes urbaines. Il est ainsi reconnu comme le lieu le plus démocratique et le plus significatif de la ville, dont «la valeur d'usage est considérée comme la principale valeur à identifier par une double lecture des usages, du passé et de l'avenir $»^{48}$. Dès 1985, l'architecte britannique Norman Foster commence par redonner toute sa grandeur au forum central en «recréant un espace public, finement traité avec de la pierre posée à la manière des Romains $»^{49}$. Peu de temps après, entre 1986 et 1987, le square de la Couronne (voir fig. 1 - réf. 17) (fig. 4, fig. 5), situé en bordure de l'Écusson ${ }^{50}$, réaménagé par les architectes et paysagistes Christine Dalnoky (née en 1956) et Michel Desvignes (né en 1958) «redevient un pôle d'animation et de convivialité $»^{51}$. 
Figure 4

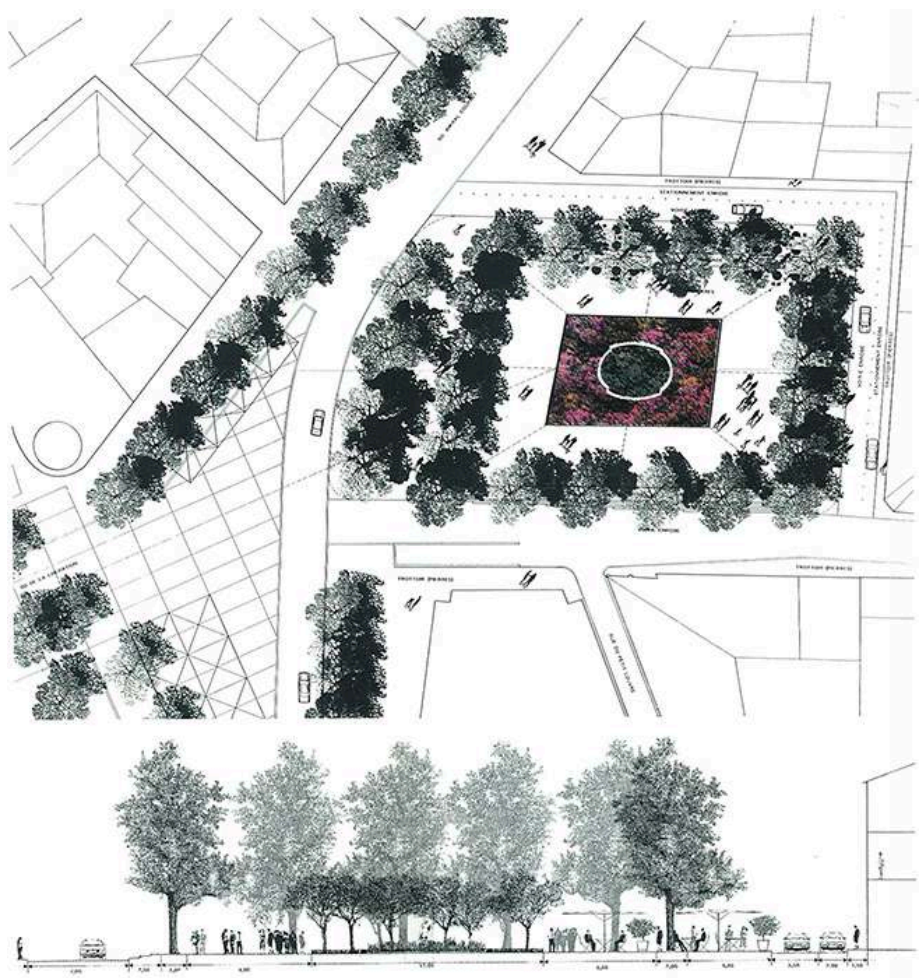

Plan de projet pour le square de la Couronne (arch. : Christine Dalnoky, Michel Desvignes, 1993), coll. Agence d'urbanisme de Nîmes.

(c) Agence d'urbanisme de Nîmes. 


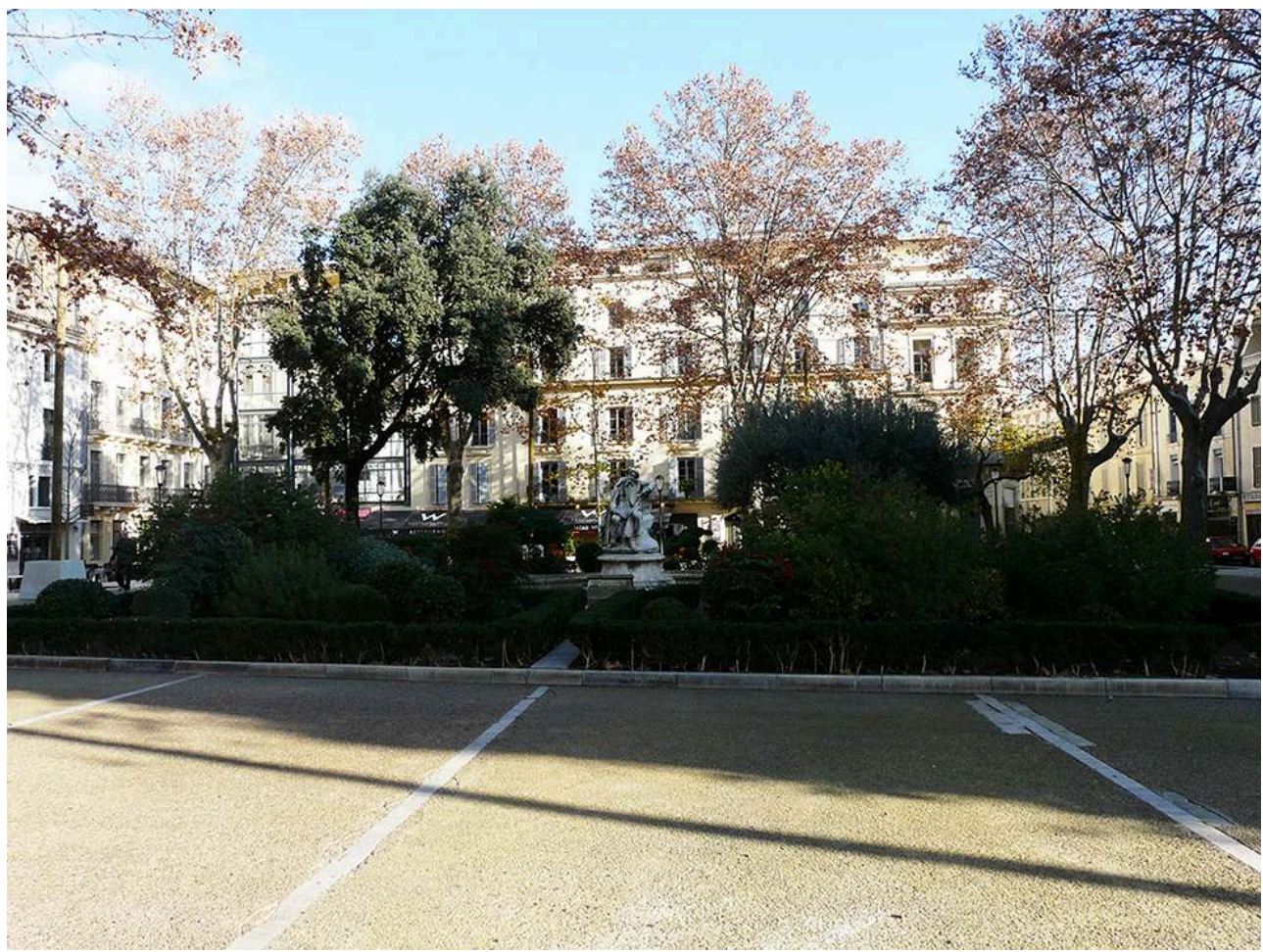

Square de la Couronne (arch. : Christine Dalnoky, Michel Desvignes, 1993).

Phot. Fanny Bocklandt, 2016. (c) Fanny Bocklandt.

11 De la même manière, la réalisation de la place Fléchier (voir fig. 1 - réf. 18), à proximité de la récente université Vauban, crée un nouvel espace public dans un quartier au tissu très dense, tout comme la rénovation de la place des Esclafidous et de l'avenue Feuchères (1993, fig. 1 - réf. 15 et 20), très fréquentée car siège de nombreuses institutions. La mise en valeur des quais de la Fontaine (1992, arch.: agence d'urbanisme de l'agglomération nîmoise, fig. 1 - réf. 21) ou encore de la place Montcalm (1992, arch.-urb. : Yves Lion, fig. 1 - réf. 19) dont le grand concours avait été remporté en 1991 par l'architecte et urbaniste Yves Lion (né en 1945) participent de cette même dynamique de requalification urbaine.

Nous avons commencé par le traitement de la place de la Maison Carrée, et chacun a pu apprécier le résultat. Nous poursuivons actuellement ce travail pour l'étendre, en partant du centre, à toute la ville. Ce faisant, on s'aperçoit que tout est à reprendre : la voirie, le mobilier urbain, les espaces verts ${ }^{52}$,

déclare ainsi Jean Bousquet dans Le Moniteur Architecture en 1994. Si l'espace public nîmois constitue désormais une armature qualifiante qui renforce l'identité des quartiers et recrée les conditions d'une vie agréable, il est aussi le lieu de rencontres de disciplines aussi diverses que l'architecture, le design, l'aménagement paysager ou l'art.

En effet, Jean Bousquet invite des artistes plasticiens à travailler à Nîmes, de Nikki de Saint-Phalle ${ }^{53}$ à Éric Watier ${ }^{54}$ (voir fig. 1 - réf. A et J), à s'exprimer au cœur de la ville, avec l'idée que l'art, au même titre que les autres arts de l'espace tels que l'architecture et l'urbanisme, participent intimement à l'art urbain, à l'art de bâtir des villes ${ }^{55}$. Jean Bousquet aspire en effet à bâtir avec art, à retrouver l'art urbain des grandes périodes historiques ${ }^{56}$. L'exemple de Barcelone, ville qui, elle aussi, part dès le début des années 1980 à la reconquête de son domaine public, a une influence déterminante sur la 
stratégie d'interventions artistiques mise en œuvre à Nîmes, tout comme les expériences conduites en France à Nantes ou à Grenoble ${ }^{57}$. À Nîmes, des artistes, représentant différents courants, invités ou ayant répondu à un appel à candidature de l'agence d'urbanisme, sont assez systématiquement associés à la conception de l'espace public, selon une méthode pragmatique et concrète : les œuvres ont pour fonction de ponctuer le parcours du citoyen ou du visiteur, de signaler un lieu de rendez-vous ou, plus simplement, d'embellir la ville. La présence d'« œuvre[s] d'art dans la rue » signifie aussi que l'art "va à la rencontre de tous", argument politique mis en avant pour valoriser l'action de Jean Bousquet.

L'idée qui prévaut est bien «d'agir par des réalisations ponctuelles qui marquent l'espace comme autant de bornes territoriales, comme l'avaient fait les urbanistes de Ferrare au XVI $\mathrm{e}^{\mathrm{e}}$ iècle $\aleph^{58}$, dans le but de renforcer l'identité des quartiers et de rendre la ville accessible à tous. Cette démarche expérimentale et ce processus conceptuel guident le réaménagement des boulevards ceinturant l'Écusson et conduisent à la réalisation d'un "musée éclaté » dont la collection est "dispersée » dans l'espace urbain. Les œuvres sont le fait d'artistes et de designers qui travaillent en association avec les architectes et les urbanistes. Martial Raysse (né en 1936), artiste peintre et sculpteur, propose une véritable expérience philosophique en investissant la place d'Assas (voir fig. 1 - réf. 14) par une scénographie autour du thème de la fontaine (1986) (fig. 6) ${ }^{59}$.

Figure 6

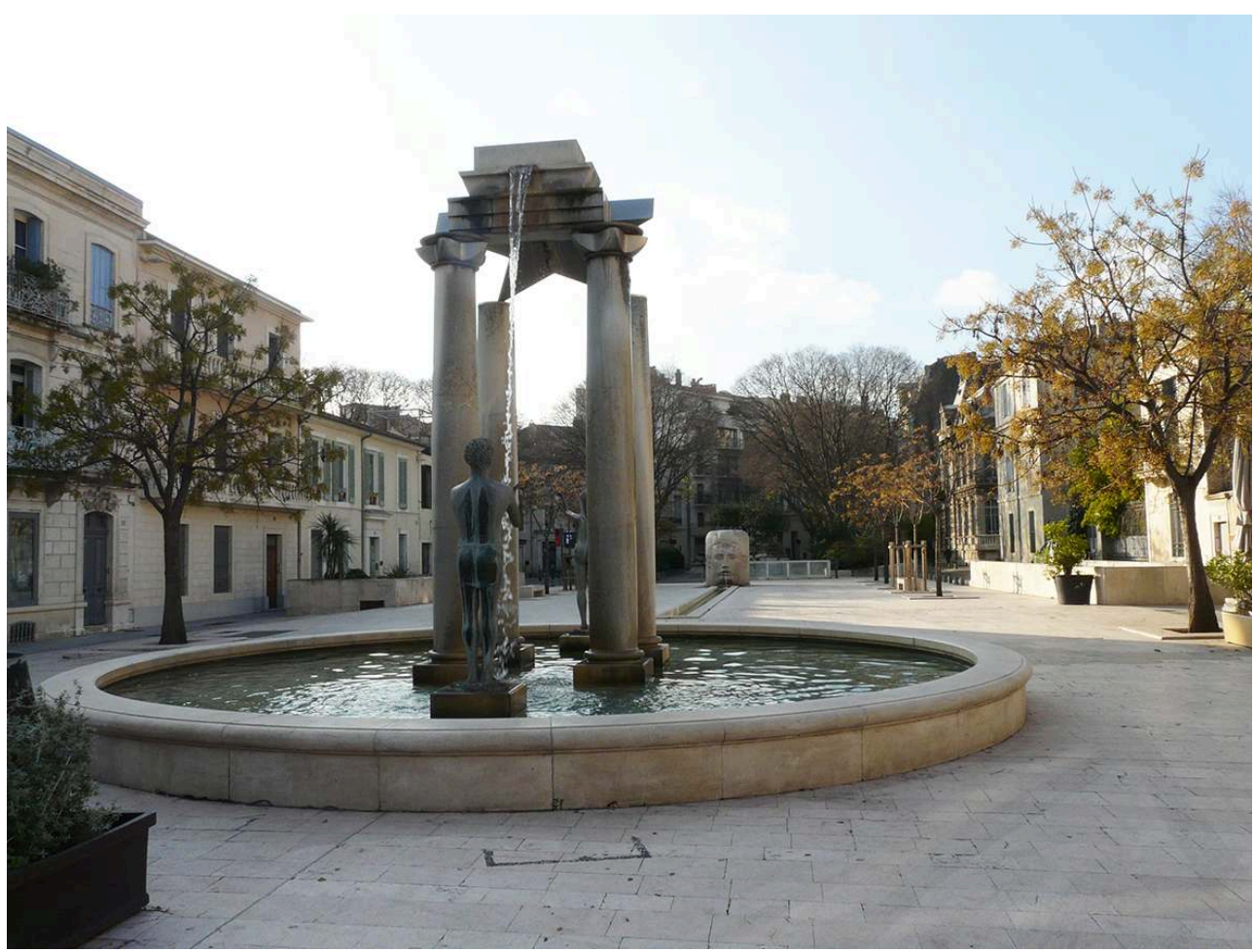

Place d'Assas (art. : Martial Raysse, 1986).

Phot. Fanny Bocklandt, 2016. (c) Fanny Bocklandt.

Il implante également sur la place du Marché (voir fig. 1 - réf. 16) redessinée par Philippe Starck sa Fontaine au Crocodile (1989, (voir fig. 1 -réf.D), qui s'impose rapidement comme un signal, comme un point de rencontre. D'autres "signaux» 
artistiques fleuriront un peu partout : sculptures monumentales de Bernard Pagès (né en 1940) (voir fig. 1 - réf. H) ou de Takis (né en 1925) (voir fig. 1 - réf. E) (fig. 7), œuvre murale de Philippe Favier (né en 1957) (voir fig. 1 - réf. F) ou fontaine d'Erik Dietman (1937-2002) (voir fig. 1 - réf. G) ${ }^{6}$. Notons cependant que ces collaborations semblent rapidement se limiter à leur seule intention théorique, les initiatives de chacun se contentant de cohabiter sans parvenir à créer une véritable œuvre collective. Si elles n'ont donc pas toujours donné lieu à la synergie créatrice qui était escomptée, il n'en demeure pas moins que ces incursions de l'art dans l'espace urbain constituent une matrice à partir de laquelle la ville s'est enrichie et développée. Elles sont les premières matérialisations de l'art de la ville qu'entend atteindre Jean Bousquet ${ }^{61}$.

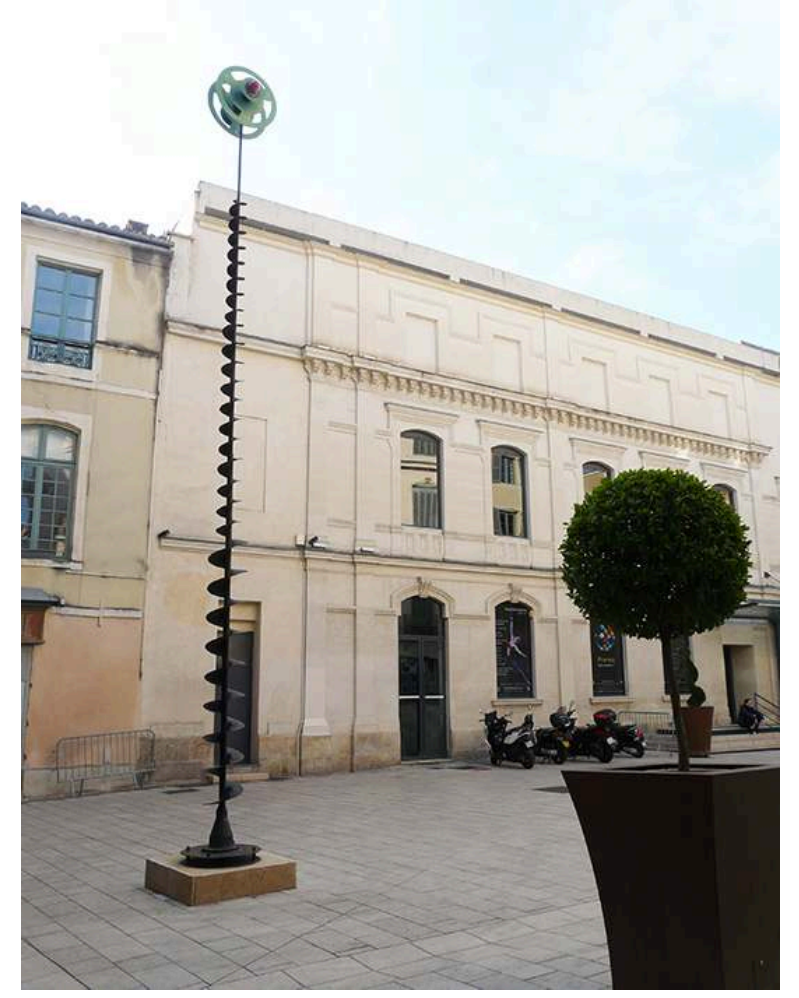

Le Signal (art. : Takis, 1989).

Phot. Fanny Bocklandt, 2016. (c) Fanny Bocklandt.

\section{Un projet collectif à l'épreuve des processus de réalisation}

Rapidement, ces différentes interventions vont pourtant faire apparaître des difficultés inhérentes à la complexité du territoire nîmois et des réseaux d'acteurs qui y interviennent. En effet, si les projets de développement concernent la seule ville de Nîmes, ils renvoient encore à des secteurs urbains dont les fonctionnalités et les spécialisations restent indépendantes les unes des autres. Leur gestion n'est pas du seul ressort de la municipalité mais fait aussi intervenir l'État et les instances territoriales ${ }^{62}$. La multiplicité des acteurs et les logiques hiérarchiques rendent difficiles l'émergence et la mise en œuvre d'un projet cohérent. Jean Bousquet propose de dépasser ces 
difficultés, qui ne sont pas spécifiques à Nîmes, en mettant en œuvre une gestion entrepreneuriale de la Ville :

Dans une entreprise, une décision de la direction est instantanément transmise aux employés pour exécution; dans une mairie, on est tributaire de plusieurs administrations, celle des ministères et la nôtre. On perd trois à six mois entre la prise de décision et la réalisation. La décentralisation n'a pas gommé cela ${ }^{63}$.

Partant de ce constat, il estime fondamental « d'affronter les logiques administratives qui tendent à faire rendre la gestion du territoire urbain tributaire d'une mosaïque d'unités décisionnelles étrangères les unes aux autres, où la pensée demeure cloisonnée et solitaire $»^{64}$.

Dès sa création en 1991, l'agence d'urbanisme de Nîmes est envisagée par Jean Bousquet comme un organe de réflexion et de décision capable de déjouer les logiques sectorielles responsables, selon lui, du morcellement de la ville contemporaine. Le travail de l'agence doit aussi permettre de s'affranchir de toute pensée dichotomique dont le maire estime qu'elle isole chacune des étapes du projet urbain, depuis la programmation jusqu'à sa mise en œuvre. S'appuyant sur les nouveaux pouvoirs qui lui sont octroyés, Jean Bousquet décide ainsi de mettre en œuvre une politique que l'urbaniste municipal Nicolas Soulier qualifie de "novatrice ${ }^{65}$ ", fondée sur une recherche d'une "qualité ${ }^{66}$ ", concernant l'architecture et l'espace public, le centreville comme les territoires périurbains ${ }^{67}$. Cet objectif de "qualité totale» peut être comparé à celui de "qualité zéro défaut» que l'on rencontre dans le monde de l'industrie et que Jean Bousquet expérimente au même moment dans son entreprise. Au nom de cette aspiration à une "qualité totale ", il met donc rapidement en place une "politique de créateurs $^{68}$ " et compose les équipes de travail selon une logique pluridisciplinaire.

Son parti pris est de choisir des architectes et des artistes reconnus pour leur talent ${ }^{69}$, parfois de réputation internationale. Il les invite à œuvrer seuls ou en association avec des créateurs locaux. Dans ce contexte, des architectes tels que Norman Foster, Jean Nouvel et Jean-Michel Wilmotte participent à la transformation de la ville; d'autres comme Kisho Kurokawa ou Aldo Rossi, en arrière-plan, jouent plutôt un rôle de fairevaloir, leur créativité étant appelée en renfort auprès des groupes économiques et financiers qui utilisent leur image pour vendre plus facilement les projets ${ }^{70}$. Dès 1983, il n'est donc pas une création nîmoise où la municipalité n'implique pas les plus grandes signatures nationales et internationales de l'architecture et du design. Christian Liger, conseiller municipal, le souligne dès 1990: «il y a là, les projets des bâtisseurs internationaux; les meilleurs [...]. Ceux qui bâtissent New York, Tokyo ou Bombay ; et qui ont accepté d'affronter l'incomparable bâtisseur de la Maison Carré. Il était

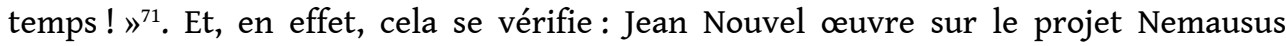
(1986), Andréa Bruno (né en 1931) travaille à la reconvention du fort Vauban en université (1995), Jean Balladur (1924-2002) réalise le parc scientifique et technique Georges-Besse (1988, voir fig. 1), Jean-Pierre Lenclos et Ariel Balmassière sont responsables de l'étude en vue de la création du secteur sauvegardé (1985) (voir fig. 1), sans oublier les multiples interventions de Jean-Michel Wilmotte ${ }^{72}$ et de Philippe Starck ${ }^{73}$, ou encore le stade des Costières (1989) (voir fig. 1 - réf. 27), réalisé par Vittorio Gregotti (né en 1927). Il en va ainsi du mobilier urbain et de la voirie piétonne au coloris des façades. La presse spécialisée exulte: "Aldo Rossi en personne, s'enthousiasme Denis Picard dans Connaissance des arts, l'idole de toutes les écoles d'architecture viendra implanter un hôtel de luxe, débarrassant l'hôtellerie de Nîmes 
du modernisme "cheap" des architectures standards et autoroutières des compagnies américaines $\aleph^{74}$. Architectes, sculpteurs, peintres et designers sont impliqués dans l'élaboration du mobilier urbain: Andrée Putman (1925-2013) réalise une corbeille (1988), Philippe Starck un abribus (1987, (voir fig. 1 - réf. C) (fig. 8), Norman Foster un panneau publicitaire (1988) tandis que Jacques Anziutti (né en 1953) dessine le mât des luminaires du centre-ville (1988).

Figure 8

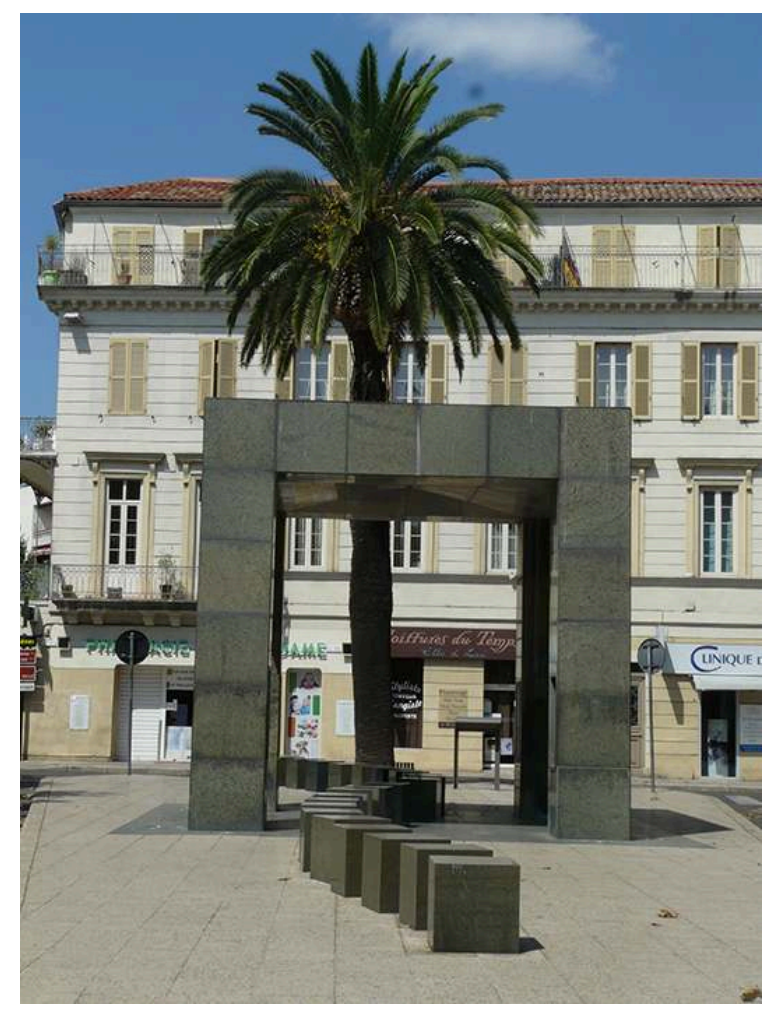

Abribus (arch. : Philippe Starck, 1987).

Phot. Fanny Bocklandt, 2016. (c) Fanny Bocklandt.

Il est légitime de se demander si cette action n'a pas conduit, en définitive, à une création "hors les murs ", négligeant les professionnels et les artistes nîmois? Les collaborations entre personnalités extérieures et locales reposent en effet souvent sur des confrontations et des rapports de force. Comment ces œuvres ont-elles pu s'inscrire dans une dynamique de création collective si des conflits d'intérêts étaient à l'œuvre dès les prémices du processus de création? Jean Bousquet estime que

lorsque Jean Michel Wilmotte crée le design des nouveaux bureaux de la mairie, ou Philippe Starck l'aménagement des studios de Radio Nemo, cela provoque de la visibilité dont les artistes nîmois profitent. [Et de rajouter] La vie d'une ville, ce n'est pas de se refermer sur soi. C'est savoir accueillir, échanger, produire avec d'autres. La culture ne doit pas être intimiste ${ }^{75}$.

21 Il est vrai que pendant douze ans, la politique architecturale du maire de Nîmes engendre des retombées au niveau local. Les tensions qui auraient pu être induites par le "parachutage » de praticiens reconnus se soldent par une émulation jugée quasi unanimement comme bénéfique par les maîtres d'œuvre et les maîtres d'ouvrage locaux. Emilio Donato est consulté pour la conception du boulevard périphérique sud (voir fig. 1 - réf. 23) en vertu de la réputation qu'il a acquise en Catalogne, sa région 
d'origine, grâce à des projets tels que la Route des eaux (1982). L'intervention de cet architecte, peu habitué aux décompositions décisionnelles territoriales, professionnelles et culturelles qui règnent en France ${ }^{76}$, revêt un caractère à la fois dynamique et prometteur : «son savoir est un atout pour l'enseignement $»^{77}$ met ainsi en avant Nicolas Soulier. Ainsi, au-delà de la dimension «promotionnelle » du choix de concepteurs étrangers, souvent internationalement reconnus, cette ouverture participe d'une réelle volonté de renouveler l'architecture et la ville qui, dans une certaine mesure, aboutit ${ }^{78}$.

\section{Traces et significations des œuvres " collectives " dans l'espace d'hier et d'aujourd'hui}

Il semble cependant exister une tension, voire une incompatibilité entre un «urbanisme de prestige» souhaité par la municipalité Bousquet et des actions plus quotidiennes. En choisissant de pratiquer une politique basée sur le développement d'une image de marque, la municipalité de Jean Bousquet exclut - en dépit de ses déclarations de principe - une partie de la ville de son développement. En effet, dans un contexte général qui confère aux villes une valeur marchande, les images produites par l'intervention de petits groupes d'initiés sont souvent en décalage avec certaines des réalités urbaines. Comme l'analyse André Chantal, dans le monde occidental, "on assiste à une sorte de déréalisation de l'espace urbain, le discours et l'image, autrement dit l'effet de réel, supplantant la réalité immédiate $»^{79}$. À l'instar d'autres villes, l'image de Nîmes fabriquée et diffusée par la municipalité ne correspond pas toujours au cadre de vie quotidien de la majeure partie de la population. La mairie, érigée en entreprise, est devenue « une structure performante fondée sur l'idéologie de la réussite et sur l'élimination des contraintes sociales $»^{80}$. Mais, le projet urbain "Expérience Nîmes " laisse par exemple entière la question des grands ensembles périphériques des années 1950 et 1960 qui apparaissent comme des zones « non signifiantes » de l'espace urbain. Il en est ainsi de la ZUP Pissevin-Valdegour (1960-1983, arch. : Xavier ArsèneHenri) et plus généralement de tous les quartiers, ou groupes de quartiers, situés derrière ou en dehors de la "vitrine » du centre-ville, qu'il s'agisse des faubourgs surplombant au nord le centre historique, des quartiers périphériques du Mas-deMingue ou de ceux du Chemin-bas d'Avignon (voir fig. 1) (fig. 9). 


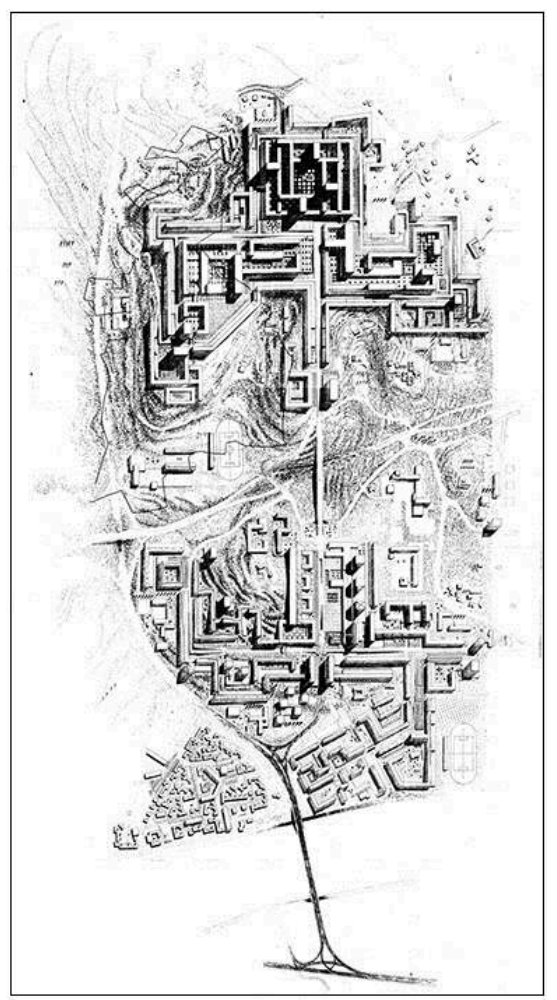

Plan de la ZUP avec, au nord, le quartier de Valdegour et, au sud, le quartier de Pissevin (arch. -urb. Xavier Arsène-Henri, 1960). Archives de la Ville de Nîmes, Série RU 82.

(c) Archives de la Ville de Nîmes.

Dès lors, comment penser la cohérence qui fonde l'unité de la cité lorsque le projet urbain lui-même semble nier les grandes modifications du xxe siècle constituées par les grands ensembles périphériques, et notamment la ZUP Pissevin-Valdegour? Pour certains observateurs, cette ZUP est pourtant «la seule vraie rupture ${ }^{81}$ » de l'espace urbain nîmois, mais dans le discours municipal elle sera plutôt qualifiée comme "un étrange espace ${ }^{82}$ » dont le projet de réinsertion passerait avant tout par le renforcement de la desserte collective. En 1991, l'image de la ZUP de Nîmes demeure donc celle d'un problème dont on n'imagine pas vraiment l'issue. Malgré la volonté affichée d'un projet urbain conçu pour « unifier une ville morcelée ${ }^{83}$ ", la redéfinition d'une économie basée sur le développement touristique et culturel contribue, à Nîmes, au renforcement des déséquilibres en créant de véritables « délaissés » urbains. Il est vrai que, d'une manière plus générale, «l'image de la ville », forgée par la société qui l'habite, a souvent été écartée par les décideurs, ou simplement laissée au domaine de l'art et de la littérature. Néanmoins, pour André Chantal, nombreux sont les observateurs qui, au début des années 1990, remarquent la force de cet imaginaire dans la reconstruction permanente de la ville ${ }^{84}$. L'évolution de l'image des grands ensembles par exemple, depuis celle d'une «ville moderne» vers celle d'une «ville déshumanisée ", n'explique bien sûr qu'une part de l'échec de cette politique, mais personne ne songerait à en contester l'importance. Ainsi, remarque-t-on à Nîmes, dans les dernières années du second mandat de Jean Bousquet, le travail de l'agence d'urbanisme s'oriente-t-il vers la prise en compte des grands ensembles dans la logique urbaine globale. 
Divers projets artistiques sont alors lancés pour requalifier cet « étrange espace ». L'un prévoit par exemple que les voitures puissent bientôt « circuler sur des rampes posées sur pilotis décollées à la fois des immeubles et du sol » ${ }^{85}$. Le programme « Banlieues $89^{86}$ ", conduit à Nîmes à partir de 1991 par un groupe d'architectes locaux en collaboration avec l'agence d'urbanisme, vise à « réinsérer la ZUP à la ville en recréant des liaisons par la réalisation d'interventions paysagères $\|^{87}$ qui sont confiées à l'architecte Gérard Grandval (né en 1930). Un renforcement de la desserte collective est permis par un nouveau plan de circulation mis en place en 1993 mais il n'en demeure pas moins que c'est explicitement une des œuvres métallo-textiles de la designer Marie-Christine Dorner (née en 1962) qui, symboliquement, doit venir affirmer la centralité et l'identité du quartier (voir fig. 1 - réf. B et B ${ }^{\prime 88}$ (fig. 10). En effet, certains éléments de la nouvelle structure urbaine, comme les quatre principales stations de bus, devront être traitées de façon similaire dans tous les quartiers. Pensées comme des lieux d'échanges, ces "modules métallo-textiles sur plan carré en série [abriteront] un kiosque de vente et les usagers de la pluie ou du soleil. [...] Un ruban fonctionnel de mobilier en lames de bois d'ipé avec bancs, fontaines d'eau potable, téléphone et éclairage y sera intégré ${ }^{89}$.

Figure 10

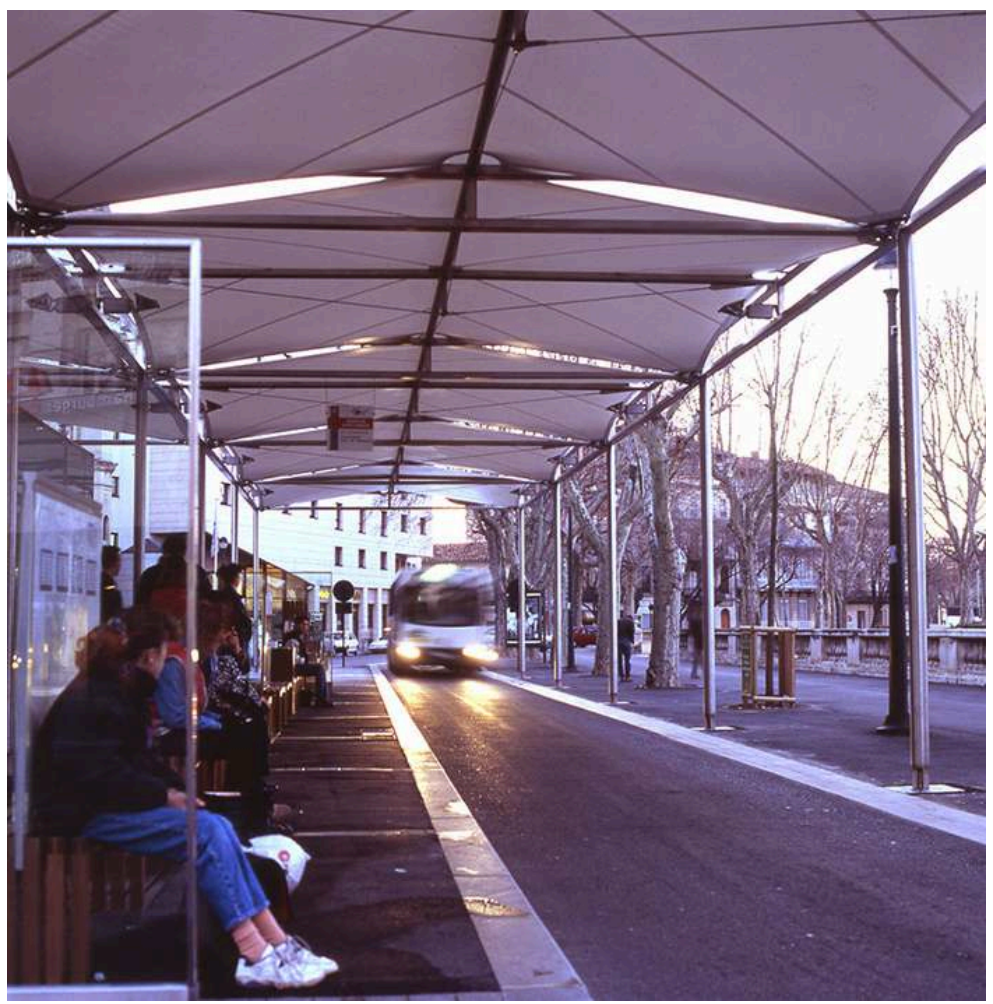

Station de bus Esplanade (art. : Marie-Christine Dorner, 1993).

Phot. Marie-Christine Dorner, 1993. (c) Marie-Christine Dorner.

À Nîmes, les réflexions engagées sur l'espace public au cours des années 1983-1995 reposent donc sur l'idée que «l'art [sous toutes ses formes] est un vecteur d'appartenance et d'identité $\aleph^{90}$. Mais cette affirmation de Jean Bousquet peut être mise en regard avec les analyses d'architectes et d'historiens qui, à l'instar de Daniel Le Couëdic, considèrent que " remiser la technicité, renoncer au court chemin d'une cause supposée à ses effets présumés, s'en remettre à l'imprévu d'une création artistique : ne 
serait-ce pas abandonner le totalitarisme technocratique pour un obscurantisme guère plus rassurant? $\|^{91}$. Le philosophe Hegel, déjà, avait mis en garde contre une confiance excessive que l'on accorderait au pouvoir de médiation de l'art. À cette capacité supposée, il avait posé un préalable : «Que la forme artistique ne [soit] pas un simple ornement destiné à agrémenter un enseignement abstrait, mais que le contenu ne fasse qu'un avec la forme figurée et que cette unité constitue son côté essentiel $»^{92}$. En effet, si la création artistique participe au développement de l'esprit critique et de la sensibilité personnelle en ouvrant de nouvelles perspectives sur le monde, elle n'est pourtant pas à la portée de tous, ce qui rend son enseignement primordial. Cette "nécessité » didactique n'échappe pas à Jean Bousquet. Il tente de la favoriser en réaménageant l'école d'art de Nîmes (1987, arch.: Ariel Balmassiere) mais aussi en créant de nouvelles filiales d'art et de design à l'université Vauban à la rentrée de septembre 1995, ou encore un atelier de design industriel et trois ateliers d'artistes pour permettre à de jeunes créateurs de travailler in situ.

Le projet urbain "Expérience Nîmes ", dans sa finalité, ne s'apparente pas vraiment à une œuvre collective mais plutôt à une somme d'interventions réparties dans la ville. Pourtant, l'art et l'architecture ont bien été invités à dialoguer dans l'espace de la cité. Aujourd'hui, certaines œuvres ont été détruites ${ }^{93}$, d'autres déplacées ${ }^{94}$ mais la plupart font toujours partie d'un paysage devenu familier où elles voisinent avec des œuvres plus récentes. Dans cette ville qui a obtenu le label « Ville d'art et d'histoire » en 1985 se pose désormais la question de la dimension patrimoniale des œuvres, des espaces et des architectures produites dans le cadre du projet urbain «Expérience Nîmes ». Une nouvelle échéance, concernant les enjeux de développement touristique et culturel à venir, semble fournir un élément de réponse : la candidature de Nîmes à l'inscription au Patrimoine mondial de l'Unesco à l'horizon 2018. Envisagée depuis 2001, cette candidature n'a été définie précisément qu'en 2011, année au cours de laquelle le comité scientifique a identifié un thème - "Nîmes, l'Antiquité au présent " $^{95}$ - et a choisi de mettre en avant « l'unicité » du patrimoine nîmois et la valeur universelle de la romanité de la ville ${ }^{96}$. Comme depuis les années 1980 les périmètres d'inscription de l'Unesco se sont agrandis ${ }^{97}$, la Ville espère voir classer au Patrimoine mondial de l'Unesco un territoire urbain de plus de 100 hectares, délimité par les jardins de la Fontaine, au nord-ouest, incluant le quartier de l'Écusson et, au sud, les environs immédiats de l'avenue Feuchères jusqu'à la gare (voir fig. 1). D'ici à 2018, la ville de Nîmes pourrait donc bien, de fait, réaffirmer l'importance de sa riche histoire architecturale et urbaine dont le projet "Expérience Nîmes" constitue l'un des derniers jalons. Lorsque le musée de la Romanité sera achevé (concours en 2011, réalisation en cours, livraison prévue pour 2018, arch.: Christian et Élisabeth de Portzamparc) (voir fig. 1 - réf. 12), en 2018, ce seront trois lauréats du prix Pritzker Norman Foster, Jean Nouvel et Élisabeth de Portzamparc - qui auront contribué à façonner le visage du "présent » - c'est-à-dire du passé récent - mis en avant dans la candidature nîmoise, mobilisant chacun à leur manière l'héritage antique de la ville ${ }^{98}$. 


\section{NOTES}

1. - BOUSQUET, Jean. « Jean Bousquet. Interview ». Dans LETESSIER, Joëlle. « L'architecture est à Nîmes ». Architecture intérieure-CREE, hors série, 1986, p. II-XXVII.

2. - VIVANT, Elsa. Qu'est-ce que la ville créative ? Paris : Puf, coll. « La ville en débat », 2009.

3. - Loi relative aux droits et libertés des communes, des départements et des régions, $\mathrm{n}^{\circ} 82-213$, 2 mars 1982, dite loi de décentralisation du territoire français.

4. - BIAU, Véronique. L'architecture comme emblème municipal. Les grands projets des maires. La Défense : PUCA, 1992.

5. - HAYAT, Yves. «Éditorial ». Galeries Magazine, n¹6, 1986, p. 49-57.

6. - Georges Frêche, appelé aussi «le bâtisseur», fut élu maire de Montpellier en 1977 et le restera jusqu'à son décès en 2004. Il fut à l'initiative de nombreux projets comme le "Corum ", centre de congrès et opéra, ou le quartier "Antigone »; Pierre Mauroy, premier ministre de François Mitterrand entre 1981 et 1984, fut maire de Lille de 1973 à 2001. Les Lillois lui doivent de grandes réalisations dont le projet " Euralille », quartier d'affaires reconnu au niveau européen ; Raymond Barre, quant à lui, fut maire de Lyon de 1995 à 2001. Il fut l'instigateur d'un concours international, lancé en 1997 pour le projet "Confluence », ayant pour but de doubler la superficie du centre-ville de Lyon; Alain Chénard, maire de Nantes de 1977 à 1983, lança pour sa part une politique ambitieuse de transports publics en réintroduisant le tramway dans la ville. Les Nantais lui doivent également la construction de la médiathèque Jacques-Demy ou du stade de la Beaujoire. Enfin, Jean Royer, ministre du gouvernement de Pierre Messmer II et maire de Tours de 1959 à 1995, promut l'arrivée du TGV ou la construction du centre de congrès Vinci.

7. - DURAN, Jacques-Olivier, HENRY, Guy. Vers une ville sans banlieue: expérience Nîmes. Paris : Éditions du Centre Pompidou, 1993.

8. - JUVIN, Joseph, SOULIER, Nicolas. La qualité de l'architecture, l'art dans la ville à la qualité de la ville, l'art de la ville. Document non publié, Agence d'urbanisme et de développement de la région nîmoise. Nîmes : 26 août 1991.

9. - Cacharel est une entreprise de prêt-à-porter fondée à Paris en 1958.

10. - L'ESTANG, Philippe de. "Jean Bousquet: donnons aux créateurs les moyens de leur création ». Galeries Magazine, nº16, 1986, p. 49-57.

11. - BOUSQUET, Jean. «Entretien ». Dans GUEZ, Jean-Paul, VERDIER, Thierry. " Nîmes en projets ». Architecture méditerranéenne, hors série, 1989, p. 139-169.

12. - Architecture Méditerranéenne est une revue semestrielle internationale consacrée principalement à l'architecture et aux beaux-arts sur le pourtour du bassin méditerranéen. Éditée en France, à Marseille plus précisément, la revue fut créée en 1968. Elle paraissait alors deux fois par an et était distribuée par abonnement aux architectes et maîtres d'ouvrage. Elle était également disponible dans les grandes librairies du Maroc.

13. - L'ESTANG, Philippe de. Art. cit.

14. - À cette époque, les "grands travaux » du président François Mitterrand marquent à leur manière dans l'histoire une nouvelle étape de la mise en représentation de la cité. Ses projets, qui concernaient tous les domaines de la culture, de la musique à la lecture en passant par les arts plastiques ou le savoir scientifique et technique, situés en Île-de-France et en province, allaient lui offrir « une occasion unique de laisser son empreinte dans l'histoire » (CHASLIN, François. Les Paris de François Mitterrand. Histoire des grands projets architecturaux. Paris : Gallimard, 1985).

15. - BOCKLANDT, Fanny. Reconversion du fort Vauban de Nîmes (1687-1995). Mémoire de master dirigé par Valérie Nègre, École nationale supérieure d'architecture de Paris-la Villette, janvier 2015. 
16. - LEONI, Giovanni. Norman Foster. Arles: Actes Sud, 2008 ; PICARD, Denis. «Carré d'Art et urbanîme ». Connaissance des arts, $\mathrm{n}^{\circ}$ 495, mai 1993, p. 52-63.

17. - JODIDIO, Philip. Jean Nouvel. 1945. Donneur de formes. Cologne : Taschen, 2012.

18. - FAYOLLE, Albane, VOURC'H, Anne, NATALI, Jean-Marc. Expérimentations. Des logements à grandes surfaces. Document non publié, Agence d'urbanisme et de développement de la région nîmoise. Nîmes, 1988. (mais des mêmes auteurs et avec un titre voisin, il existe un document publié en 1990).

19. - GOULET, Patrice. « Nîmes. Concours pour la médiathèque et le Centre d'art contemporain, octobre 1984. Norman Foster, Frank Gehry, Jean Nouvel ». AA, nº 236, décembre 1984, p. 61-69.

20. - LETESSIER, Joëlle. « L'architecture est à Nîmes ». Art. cit.

21. - Le projet de l'Atelier de Nîmes a été lancé au printemps 1984. La sélection définitive de l'équipe a été connue en décembre de la même année, ainsi que la nomination de Bernard Heurtevin à la tête des Ateliers. Martine Bedin, Gérard Barrau, Ross Lovegrove et Philippe Starck, quatre représentants «brillants » des jeunes générations de la création française, sont devenus « les » designers de Nîmes. L'architecte Jean Nouvel complète logiquement l'équipe de designers puisque selon le souhait de Jean Bousquet, « l'industriel qui viendra à l'Atelier faire redessiner un cendrier pourra repartir avec une usine». L'objectif était alors de faire de la référence «De Nîmes »: "un label équivalant à ce qu'ont été dans le monde du design des entités comme le Bauhaus, Vienne, ou Ulm... [...]. Il y aura ainsi, par voie de conséquence, autour de l'Atelier, une véritable école de Nîmes, de rayonnement international ». Centre d'animation permanent, l'Atelier devait devenir un cadre de formation au design pour les responsables d'entreprise, un lieu de séminaire où accueillir des stagiaires-résidents affectés à des recherches particulières. Philippe Starck fut, pour la première année de fonctionnement de l'Atelier, président d'un conseil de surveillance soucieux de la cohérence et de la dynamique du projet. Il eut également en charge la première commande de l'Atelier, passée par la ville de Nîmes pour son mobilier urbain. Le premier «banc lumineux en aluminium » fut ainsi bientôt installé autour des troncs des platanes des mails nîmois (Ibid.).

22. - « Depuis quelques années déjà, Nîmes s'est engagée dans un processus de transformation profond de son espace urbain par des opérations d'architecture et d'urbanisme marquantes. De nombreux professionnels, architectes, urbanistes et spécialistes divers, participent directement à cette action et un public averti de chercheurs s'intéresse à cette évolution [...]. Aussi, le sentiment que Nîmes devrait être un site d'expérimentation urbaine s'est conforté et progressivement un projet s'est formalisé sous le titre de Centre d'expérimentation de Nîmes » (JUVIN, Joseph, SOULIER, Nicolas. Document cité).

23. - Ibid.

24. - Ibid.

25. - Jean Bousquet (maire), Jean-Paul Fournier (adjoint au maire délégué à l'urbanisme), Joseph Juvin (directeur de l'agence d'urbanisme), Nicolas Soulier (urbaniste), Norman Foster (architecte) étant les principaux décideurs.

26. - BENEVOLO, Leonardo. Histoire de la ville. Roquevaire/Marseille : Éditions Parenthèses, 1983 ; MONDRIAN, Piet. Réalité naturelle et réalité abstraite. Dialogue publié en treize suites dans la revue De Stijl, 1919-1920. Traduit dans SEUPHOR, Michel. Piet Mondrian. Sa vie, son ceuvre. Paris: Flammarion, 1956, p. 338.

27. - BOUSQUET, Jean. Intervention de Monsieur Jean Bousquet. Document non publié, Agence d'urbanisme et de développement de la région nîmoise. Nîmes : 1991.

28. - « Les responsables de la ville ne produisent plus que des isolats. Leurs pensées, séparées les unes des autres, s'épuisent dans des réflexions solitaires, faute d'être alimentées par l'échange ou la confrontation » (DEHAN, Philippe. «Expérience Nîmes ». Le Moniteur. Architecture AMC, ${ }^{\circ}$ 26, novembre 1991, p. 35-58).

29. - L'ESTANG, Philippe de. Art. cit. 
30. - BOUSQUET, Jean. Intervention de Monsieur Jean Bousquet. Document cité.

31. - « Aujourd'hui justement notre époque est créative. On ne doit pas l'enfermer, mais la laisser s'exprimer " déclare en 1986 Jean Bousquet, dans un entretien qu'il accorde à Philippe de L'Estang pour Galeries Magazine (art. cit.).

32. - « les chercheurs [ont] trop vite oublié que la ville est un tout et que chaque fonction urbaine est intimement dépendante de l'ensemble » (JUVIN, Joseph, SOULIER, Nicolas. Document cité).

33. - Joseph Juvin est un ingénieur et urbaniste français qui a participé au début de sa carrière à l'élaboration du schéma directeur de la région parisienne et au lancement de villes nouvelles sous l'impulsion de Paul Delouvrier. Il a également contribué à la création de l'agence d'urbanisme d'Angers et de Nantes dont il a assuré la direction de 1978 à 1989. Appelé par Jean Bousquet, il a ensuite assuré à partir de 1990 et jusqu'à sa mort en 1995, la direction de l'agence d'urbanisme de la région nîmoise.

34. - Nicolas Soulier est un architecte et urbaniste français, né à Paris en 1951. Son projet le plus important, inauguré par la Ville de Paris en 1989, est l'opération Michelet, dans le $13^{\mathrm{e}}$ arrondissement de Paris. Professeur à l'école d'architecture de Versailles, en 1990 il ferme son atelier parisien pour se consacrer à la création de l'agence d'urbanisme de Nîmes, dans laquelle il travaille de 1991 à 1995.

35. - JUVIN, Joseph, SOULIER, Nicolas. Doc. cité.

36. - Ibid.

37. - Ibid.

38. - Superficie du centre historique de la ville de Nîmes: 41 hectares. Superficie de l'agglomération : 161,85 $\mathrm{km}^{2}$ (soit 16185 hectares).

39. - BOUSQUET, Jean. Séminaire sur le projet urbain de Nîmes. Intervention de J. Bousquet, député-maire de Nîmes. Document cité.

40. - « Nous avons beaucoup de savoirs et de nombreux spécialistes dans beaucoup de domaines touchant à la ville. Associons nos savoirs avec l'art de faire la ville et nous retrouverons ainsi une démarche culturelle porteuse d'espérance pour nos villes et nos concitoyens "; «La réalisation concrète nécessiterait de façon primordiale des locaux d'accueil avec les équipements d'accompagnement nécessaires » (Ibid.).

41. - Ibid.

42. - JUVIN, Joseph, SOULIER, Nicolas. Document cité.

43. - Ce phénomène, observable dans la plupart des villes françaises, a notamment été traité par l'urbaniste et géographe Marcel Roncayolo (RONCAYOLO, Marcel. La ville et ses territoires. Paris : Gallimard, 1990).

44. - TOUSSAINT, Jean-Yves, ZIMMERMANN, Monique. Projet urbain : ménager les gens, aménager la ville. Sprimont : Pierre Mardaga, 1998.

45. - JUVIN, Joseph, SOULIER, Nicolas. Document cité.

46. - Les espaces publics, et plus précisément les voies publiques, ont toujours été au cœur de domaines stratégiquement déterminants pour la ville, tels que les réseaux de transport et de circulation ou l'environnement. Une prise de conscience intervient dès le début des années 1980 et les villes commencent à redonner une plus grande importance à l'aménagement de leurs espaces et à la manière dont les habitants entrent en relation avec eux. Les réflexions engagées se traduisent par des études et des projets d'aménagement dans presque toutes les grandes villes européennes et françaises, mobilisant une part importante des budgets d'investissement des collectivités (LUCAN, Jacques. «Villes : qu'est-ce qu'un projet urbain? ». Le Moniteur Architecture, n²7, décembre 1991, p. 46-59).

47. - JUVIN, Joseph, SOULIER, Nicolas. Document cité.

48. - Ibid.

49. - Foster Associates. Place du Carré d'art. Document non publié, Agence d'urbanisme et de développement de la région nîmoise, Nîmes, 1990. 
50. - À cause de sa forme, nom donné au centre historique de Nîmes.

51. - Agence d'urbanisme et de développement de la région nîmoise, 1990-1995. Document non publié.

52. - BOUSQUET, Jean. « Jean Bousquet : donner la ville à tous ». Dans DEHAN, Philippe. « L'espace public à Nîmes ». Le Moniteur Architecture, n52-53, juin-juillet 1994, p. 28-34.

53. - Niki de SAINT-PHALLE, La chapelle, 1990, œuvre non réalisée.

54. - Éric WATIER, Coupole, 1988, 1 bd de l'Amiral-Courbet, Nîmes.

55. - «L'espace public est le lieu privilégié pour accueillir les œuvres des artistes plasticiens, sculpteurs, designers, peintres, paysagistes. [...] [Ainsi], progressivement chacun devient plutôt fier de sa ville. On lève la tête pour regarder. L'architecture et les arts plastiques deviennent familiers » (BOUSQUET, Jean. Document cité).

56. - «L'on doit dépasser la séparation de l'art et impliquer les artistes dans des expérimentations, seules capables d'infléchir la logique techniciste [...] L'art de bâtir les villes peut alors reprendre tout son sens à l'instar des grandes périodes historiques de l'urbanisme qui ont su articuler savoir et culture, comme par exemple l'époque romaine, l'époque des cathédrales, l'époque classique " déclare ainsi Jean Bousquet à Jean Jouber dans un entretien publié (JOUBER, Jean. "Jean Bousquet: À Nîmes, l'audace, ça marche! ». Écosud, octobre 1988, p. V-XXIV).

57. - En France en effet, de nombreux espaces publics ont déjà été le lieu d'interventions d'artistes qui, par les dimensions et les significations de leurs réalisations, renouvellent la fonction de l'art dans l'espace public. Cette expérience est très bien rapportée par Daniel Abadie (ABADIE, Daniel, DAVAL, Jean-Luc, DELLOYE, Charles, et al. L'art et la ville, urbanisme et art contemporain. Genève : Skira/Secrétariat général des villes nouvelles, 1990).

58. - DEHAN, Philippe. « L'espace public à Nîmes ». Art. cit.

59. - BENEL, Jean-Luc. «Martial Raysse à Nîmes. Messages cachés de la place d'Assas ». Nemausensis, En ligne. [Consulté le 05 janvier 2017]. Voir : http://www.nemausensis.com/Nimes/ PDF/MessagesCachesAssas.pdf.

60. - Bernard Pagès, Hommage à Albert Camus, place Hubert-Rouger; Takis, le Signal, place de la Calade ; Philippe Favier, Peinture murale, salle de lecture du Carré d'art, [disparu]; Erik Dietman, fontaines métalliques, [disparu].

61. - «Ces interventions, parfois très modestes, sont les premiers éléments d'une toile qui se tisse patiemment et qui, à terme, donnera corps au projet urbain de la ville » (BOUSQUET, Jean. Document cité).

62. - «L'urbain est séparé de l'économique, le social de l'économique, les institutions déconcentrées continuent de traiter les espaces de façon autonome» souligne notamment Nicolas Soulier (Bureau de la recherche architecturale. Contribution à la réflexion de l'impact du projet d'architecture sur le développement urbain: Rapport entre l'architecture et la ville: Journées d'études. Communication présentée à l'École d'architecture de Paris-Belleville, colloque, Paris, décembre 1992).

63. - BOUSQUET, Jean. Document cité.

64. - SOULIER, Nicolas. "Nîmes", [dans] Bureau de la recherche architecturale. Contribution à la réflexion de l'impact du projet d'architecture sur le développement urbain: Rapport entre l'architecture et la ville: Journées d'études. Document cité.

65. - Ibid.

66. - « Notre responsabilité est là, on pourra discuter sur les problèmes financiers, mais on ne discutera pas sur la qualité » (Ibid.).

67. - Jean Bousquet considère que cette notion de qualité « doit s'appliquer sur des secteurs géographiques suffisamment vastes pour traiter tous les aspects du sujet [la ville]» (BOUSQUET, Jean. Document cité). Il n'hésite pas à déclarer qu'« il n'y a pas d'autre possibilité pour faire une création de qualité » (Id. « Entretien ». Art. cit.). 
68. - «Face à cette responsabilité énorme qui engage l'avenir, j'ai immédiatement pressenti l'intérêt de faire quelque chose et de s'engager à fond pour vaincre la timidité française face à l'architecture, ce complexe face à l'innovation. [...] C'est à ce moment-là que j'ai décidé d'avoir une exigence de qualité architecturale et que j'ai immédiatement mis en œuvre une politique de créateurs » déclare Jean Bousquet dans l'entretien qu'il accorde à Philippe de L'Estang en 1986 (art. cit.). Cette « politique de créateurs » passe par la sélection.

69. - « Nîmes a systématiquement fait appel à des architectes de talent qui ont ajouté à la valeur d'utilité de ces lieux une valeur artistique et ont contribué ainsi à l'amélioration du cadre et donc de la qualité de vie des citoyens » (BOUSQUET, Jean. Document cité).

70. - Ces architectes «faire-valoir » interviennent dans le processus de création soit pour viser le projet dans sa phase finale, soit dans la phase d'esquisse afin de développer un concept. (fig. 1 réf. 11 et 26).

71. - LIGER, Christian. Nîmes sans visa. Portait d'une ville. Paris : Seghers, 1990.

72. - Jean-Michel Wilmotte : rénovation et aménagement du musée des Beaux-Arts (1987) (fig. 1 réf. 5) ; restauration et aménagement de l'hôtel de ville (1990) (fig. 1 - réf. 7) ; restructuration et aménagement du théâtre municipal (1988) (fig. 1 - réf. 10); aménagement extérieur des halles (1988) (fig. 1 - réf. 8) ; rénovation et aménagement de l'hôtel Le Cheval Blanc (1990) (fig. 1 - réf. 9).

73. - Philippe Starck: aménagements du centre d'action sociale (1984) (fig. 1 - réf.6); porte urbaine à l'intersection de l'avenue Jean-Jaurès et de la voie ferroviaire (non réalisée) (fig. 1 réf. I) ; aménagement des locaux de Radio Nemo (1988) (fig. 1 - réf. 13).

74. - PICARD, Denis. «Carré d'Art et urbanîme ». Connaissance des arts, nº495, mai 1993, p. 52-63 ; Aldo Rossi ne réalise en définitive que le dessin d'esquisse des façades extérieures du bâtiment (fig. 1 - réf. 11).

75. - L'ESTANG, Philippe de. Art. cit.

76. - En Catalogne, il n'existe pas les mêmes coupures professionnelles qu'en France entre architectes, paysagistes et ingénieurs.

77. - SOULIER, Nicolas. « Nîmes ». Art. cit.

78. - «C'est une très bonne chose pour l'architecture. L'expérience nous le prouve. À Nîmes aujourd'hui les gens parlent d'architecture, la regardent, lèvent la tête pour voir des façades » (L'ESTANG, Philippe de. Art. cit.).

79. - CHANTAL, André. « Changer l'image d'une ville ». Politiques et management public, vol. $5, \mathrm{n}^{\circ} 4$, 1987, p. 51-64.

80. - HOFFMANN-MARTINOT, Vincent. " Gestion moderniste à Nîmes. Construction d'une image de ville ». Les annales de la recherche urbaine, $\mathrm{n}^{\circ} 38$, juin-juillet 1988, p. 95-103.

81. - BERNIÉ-BOISSARD, Catherine. Nîmes, questions pour une ville. Lieux, enjeux, figures du social. Montpellier : Publications ARPES/université Paul-Valéry, 1995.

82. - BOUSQUET, Jean. « Savoir anticiper ». Dans DEHAN, Philippe. « Expérience Nîmes ». Art. cit., p. 35-58.

83. - DURAN, Jacques-Olivier, HENRY, Guy. Op. cit.

84. - «Cette image peut même aller très violemment à l'encontre de l'action urbanistique des autorités publiques et conduire à des échecs majeurs » (CHANTAL, André. Art. cit.).

85. - Agence d'urbanisme et de développement de la région nîmoise, 1990-1995. Document cité.

86. - Le projet « Banlieues 89 » était une mission interministérielle lancée en 1983 et qui a duré jusqu'en 1991. Animée par l'architecte Roland Castro et l'urbaniste Michel Cantal-Dupart, elle s'était donné pour but d'améliorer l'urbanisme de la banlieue en France (GUILLOT, Pascal. «Entretien avec l'architecte Roland Castro ». Cahiers d'histoire. Revue d'histoire critique. En ligne, voir le site : http://chrhc.revues.org/1925 [Consulté le 8/12/2016].

87. - Agence d'urbanisme et de développement de la région nîmoise, 1990-1995. Doc. cit. 
88. - Cette œuvre ne fut jamais réalisée. La station de bus Esplanade (fig. 10), représente une première structure qui a été construite sur l'esplanade Charles-de-Gaulle, située entre les Arènes et l'avenue Feuchères.

89. - DEHAN, Philippe. «L'espace public à Nîmes ». Art. cit., p. 28-34.

90. - BOUSQUET, Jean. Document cité.

91. - LE COUËDIC, Daniel. «Cinquante années pour faire sa place : un projet urbain en proie au temps ». Dans BOURDIN, Alain. « Atelier 1 : Projet urbain et/ou projet de ville ». Support de cours, École nationale supérieure d'architecture de Paris-Est-Marne-la-Vallée. En ligne, voir le site: http://perso.univ-mlv.fr/www-ltmu/groupe_documents/doc_pdf/resumes.pdf [consulté le 16/04/2016].

92. - DEJARDIN, Bertrand. L'art et la raison. Éthique et esthétique chez Hegel. Paris : L'Harmattan, 2008.

93. - Lors du réaménagement de la place de l'Esplanade par le cabinet d'architectes Bernard Paris \& Associé (2012), la structure métallo-textile de Marie-Christine Dorner a été détruite.

94. - La sculpture de Bernard Pagès, Hommage à Albert Camus, fut déplacée de la zone d'activité du Nouveau Nîmes vers la place Hubert-Rouger (date n. c.).

95. - Ibid.

96. - « On s'est aperçu qu'on pouvait considérer la romanité dans ce qu'elle a induit sur la ville », explique le maire actuel Jean-Paul Fournier (SOUCHE, Jean-Pierre. «L'humanité s'offre à Nîmes ». Midi Libre, $\mathrm{n}^{\circ} 25810,2016$, p. 2-4).

97. - L'institution inscrit en effet désormais des paysages, des sites culturels et non plus seulement des édifices ou ensembles d'édifices.

98. - Parmi les grands chantiers qui ont marqué la ville de Nîmes et qui ont contribué à développer une nouvelle image de la cité gardoise, on compte bien entendu le Carré d'art, dont le projet garde sa cohérence surtout à travers la relation qu'il entretient avec la Maison Carrée. Si dix-sept siècles séparent ces deux édifices, le projet illustre de plusieurs manières comment le nouveau peut être relié à l'ancien et comment il peut respecter le passé tout en évoquant sa propre époque. Pour le critique d'architecture Francis Rambert en effet: "Maison Carrée et Carré d'art se donnent habilement la réplique [...] le nouveau bâtiment, appelé à devenir lui aussi, avec le temps, un élément central du patrimoine nîmois, entretient avec l'ancien un dialogue subtil, très sensible dans l'affirmation du portique, beaucoup plus secret dans le jeu des proportions ». C'est à cela qu'aspire aujourd'hui le duo franco-brésilien, en venant se positionner face aux Arènes (RAMBERT, Francis. "Nîmes : Foster city? ». D’A. D’Architectures, no35, mai 1993, p. 11-13).

\section{RÉSUMÉS}

Au début des années 1980, les événements politiques, économiques et sociaux qui se déroulent à Nîmes (Gard) contribuent à redéfinir son système de gestion. Par la mise en œuvre d'un projet urbain baptisé "Expérience Nîmes, vers une ville sans banlieues", la municipalité de Jean Bousquet (maire de Nîmes de 1983 à 1995) cherche une nouvelle manière de penser le développement et l'image de la ville. Les responsables et les acteurs de l'urbain se fixent comme objectif d'essayer d'unifier une ville morcelée par les effets du zoning, tout en conservant l'identité des territoires qui la composent. Ils misent pour cela sur le développent quasi 
systématique de collaborations entre urbanistes, architectes, designers et artistes pour la création d'un musée à ciel ouvert, le dessin de l'espace public ou encore la restauration d'édifices patrimoniaux. Les réflexions engagées sur l'aménagement urbain reposent en partie sur l'idée de retrouver un certain "art de bâtir la ville ». Cependant, malgré une volonté de changement clairement affirmée, le travail opéré dans l'espace urbain nîmois guidé par le jeu des représentations ne permettra pas l'unification réelle de la ville, provoquant même dans une certaine mesure l'effet inverse en renforçant les déséquilibres entre le centre et sa périphérie.

At the beginning of the 1980s, the political, economic and social events which took place in the city of Nîmes contributed to a redefinition of its urban planning system. With the project baptised 'The Nîmes experiment, towards a city without suburbs', the local authority, run by Jean Bousquet (mayor of the city from 1983 to 1995) tried to find a new way of thinking about its development and about improving its image for the coming years. The goal of the planners and other players in the field was to unify a city divided by zoning whilst preserving the identity of the territories composing it. The project sought to encourage collaboration between urban planers, architects, designers and artists in order to create a sort of open-air urban museum, redesigning public spaces and restoring heritage buildings. These reflections on urban planning are based on the idea of rediscovering a kind of 'art of city-building'. However, despite a clearly affirmed will to bring about change, the work carried out in the urban space of Nîmes, inspired by received ideas of the character of different neighbourhoods, did not lead in the end to a real reunification of the city. The project even had the opposite effect of reinforcing imbalances between the city centre and the periphery.

\section{INDEX}

Keywords : Nîmes, Jean Bousquet, urban project, cultural tourism, architecture, contemporary art

Mots-clés : Nîmes, Jean Bousquet, projet urbain, tourisme culturel, architecture, art contemporain

\section{AUTEUR}

\section{FANNY BOCKLANDT}

Architecte (ENSA Paris-La Villette, 2015), titulaire d'un M2 Histoire de l'architecture (université Paris 1 Panthéon-Sorbonne, 2016) fannybocklandt@orange.fr 